\title{
Sirolimus therapy in a child with partially diazoxide-responsive hyperinsulinaemic hypoglycaemia
}

\author{
Kah-Yin Loke1,2, Andrew Sng Anjian'2, Yvonne Lim Yijuan², Cindy Ho Wei Li ${ }^{2}$ \\ Maria Güemes ${ }^{3}$ and Khalid Hussain ${ }^{3}$
}

'Department of Paediatrics, Yong Loo Lin School of Medicine, National University of Singapore, Singapore, ${ }^{2}$ Khoo Teck Puat-National University Children's Medical Institute, National University Health System, Singapore, and ${ }^{3}$ Developmental Endocrinology Research Group, Clinical and Molecular Genetics Unit, Institute of Child Health, University College London, London, UK
Correspondence should be addressed to $\mathrm{K}$ Y Loke

Email

paelky@nus.edu.sg

\section{Summary}

Hyperinsulinaemic hypoglycaemia $(\mathrm{HH})$, which causes persistent neonatal hypoglycaemia, can result in neurological damage and it's management is challenging. Diazoxide is the first-line treatment, albeit not all patients will fully respond to it, as episodes of hypoglycaemia may persist and it entails unpleasant adverse effects. Sirolimus, an mTOR inhibitor, has reportedly been successful in treating children with severe diffuse $\mathrm{HH}$, thus obviating the need for pancreatectomy. We report a girl with $\mathrm{HH}$, with a novel heterozygous $A B C C 8$ gene missense mutation (c.4154A>T/ p.Lys 1385Thr), who was initially responsive to diazoxide therapy. After 11 months of diazoxide treatment, she developed intermittent, unpredictable breakthrough episodes of hypoglycaemia, in addition to generalized hypertrichosis and weight gain from enforced feeding to avoid hypoglycaemia. Sirolimus, which was commenced at 15 months of age, gradually replaced diazoxide, with significant reduction and abolition of hypoglycaemia. The hypertrichosis resolved and there was less weight gain given the reduced need for enforced feeding. Sirolimus, which was administered over the next 15 months, was well tolerated with no significant side effects and was gradually weaned off. After stopping sirolimus, apart from hypoglycaemia developing during an episode of severe viral gastroenteritis, the capillary glucose concentrations were maintained $>3.5 \mathrm{mmol} / \mathrm{L}$, even after a $10 \mathrm{~h}$ fast. Sirolimus may have a role in the treatment of partially diazoxide-responsive forms of $\mathrm{HH}$ who experience breakthrough hypoglycaemia, but the long-term safety and efficacy of sirolimus are not established.

\section{Learning points:}

- Conventional treatment of diffuse HH with diazoxide is not always effective in controlling hypoglycaemia and can be associated with unpleasant side effects.

- Sirolimus was successfully used to abolish recurrent hypoglycaemia in partially diazoxide-responsive $\mathrm{HH}$, with resolution of unacceptable diazoxide-associated side effects.

- Sirolimus was well tolerated with no clinically significant side effects.

- Shortly after stopping sirolimus, the capillary glucose levels remained normoglycemic. 


\section{Background}

Hyperinsulinaemic hypoglycaemia (HH) from dysregulated insulin secretion of the pancreatic $\beta$-cells is an important cause of persistent neonatal hypoglycaemia. Mutations have been reported in the $A B C C 8$ and KCNJ11 genes on chromosome 11p15.1, which encode the sulphonylurea receptor 1 (SUR1) and the inward rectifying potassium channel (Kir6.1) proteins, respectively (1). Both these proteins constitute the ATP-sensitive plasma membrane potassium (KATP) channel, the regulation of which controls cell membrane polarization, which determines insulin release from the pancreatic $\beta$-cell. Inactivating mutations of the $A B C C 8$ and KCNJ11 genes result in closure of the KATP channels, leading to depolarization of the cell membrane, opening of the voltage-dependent calcium channels and insulin release. Diazoxide has been the conventional firstline treatment of diffuse $\mathrm{HH}$ as it opens the KATP channel and inhibits insulin secretion. However, diazoxide is associated with unpleasant side effects including fluid retention, blood dyscrasias, nausea and hypertrichosis.

The use of sirolimus, an inhibitor of mTOR (the mammalian target of rapamycin) as an immunosuppressant in post-renal transplant patients uncovered its association with hyperglycaemia (2). Studies of the pancreas in patients with diffuse $\mathrm{HH}$ suggest a pathogenic role in overexpression of the mTOR pathway (3). Research has demonstrated that sirolimus can effectively suppress $\beta$-cell proliferation and reduce the severity of $\mathrm{HH}$. Inhibitors of mTOR have already been successfully used to treat adults with pancreatic insulin secreting tumours (4).

In recent reports, sirolimus was efficacious and safe in children with $\mathrm{HH}$ who were unresponsive to diazoxide therapy and octreotide, thus obviating the need for near total pancreatectomy (5). We report a 3-year 3-monthold girl who demonstrated initial partial response to diazoxide, but then developed intermittent, unpredictable hypoglycaemic episodes and severe hypertrichosis with weight gain. Sirolimus, which replaced diazoxide, was successfully used in abolishing hypoglycaemia and resolving the diazoxide-associated side effects. Shortly after stopping sirolimus, the blood glucose levels remained in the normal range, except during the stress of a viral illness.

\section{Case report}

A Chinese girl was born at 36 weeks gestation by normal vaginal delivery, with a birth weight of $3.8 \mathrm{~kg}$ (+2.1 s.D.). She was hypoglycaemic within the first
$3 \mathrm{~h}$ of life, with a capillary blood glucose level of $1.6 \mathrm{mmol} / \mathrm{L}$. She subsequently developed recurrent episodes of non-ketotic hypoglycaemia requiring an intravenous $12.5 \%$ dextrose drip with a maximal dextrose concentration of $8 \mathrm{mg} / \mathrm{kg} / \mathrm{min}$ and boluses of intravenous glucagon.

Investigations performed when she was hypoglycaemic with a serum glucose level of $0.6 \mathrm{mmol} / \mathrm{L}$ (11 mg/dL) demonstrated an inappropriately elevated serum insulin concentration of $9.4 \mathrm{U} / \mathrm{L}$, with a normal serum cortisol level of $427 \mathrm{nmol} / \mathrm{L}$, a normal growth hormone concentration of $54.6 \mathrm{U} / \mathrm{L}$ and a normal metabolic screen. The clinical and biochemical diagnosis of $\mathrm{HH}$ was made. She was then commenced on oral diazoxide and transferred to our hospital at day 8 of life for continued management.

On initial assessment, she was plethoric, well thrived with mild facial hypertrichosis. Her tone was normal, with a normal Moro reflex. Over the next 3 weeks, she required increasing doses of diazoxide, reaching a maximum dose of $20 \mathrm{mg} / \mathrm{kg} /$ day, so as to maintain normoglycaemia. Hydrochlorothiazide, which has a synergistic effect in suppressing insulin secretion, was added to prevent fluid retention. She did not require further escalation of treatment to octreotide, glucagon or nifedipine. Concurrently, the glucose infusion rate was decreased as she was weaned to full feeds providing $106 \mathrm{cal} / \mathrm{kg} /$ day, with the addition of glucose polymers and cornstarch. Her initial response to diazoxide allowed her to be discharged with capillary glucose levels above $3.5 \mathrm{mmol} / \mathrm{L}$ and a fasting duration of $6 \mathrm{~h}$.

She was subsequently followed up at the paediatric endocrine specialist clinic. She experienced no hypoglycaemic episodes and was weaned off glucose polymers and cornstarch with a gradual reduction of diazoxide to a minimum dose of $9 \mathrm{mg} / \mathrm{kg} /$ day at 4 months of age. However, the frequency of intermittent borderline hypoglycaemic episodes (lowest capillary glucose level of $2.5 \mathrm{mmol} / \mathrm{L}$ ) increased to an average of twice a week from 6 months of age, necessitating a gradual increase in the diazoxide dose to $11.7 \mathrm{mg} / \mathrm{kg} /$ day.

Genomic DNA was extracted from peripheral blood leucocytes from the infant and both clinically unaffected parents using standard procedures and sent to the molecular genetics laboratory at the University of Exeter Medical School for analysis of mutations in the $A B C C 8$ and $K C N J 11$ genes. This confirmed that the infant was heterozygous for a novel missense mutation in the $A B C C 8$ gene (c.4154A>T/p.Lys1385Thr). Since the lysine residue is highly conserved, this mutation is very likely to be pathogenic. Analysis of both parents demonstrated that the mutation arose de novo and 
originated from the paternal copy of the ABCC 8 gene. Given the genetic finding, in order to exclude a focal lesion, the child subsequently had an ${ }^{18} \mathrm{~F}$-Fluoro-L-DOPA positron emission tomography scan, which confirmed that she had the diffuse form of $\mathrm{HH}$.

Subsequently, the intermittent breakthrough episodes of hypoglycaemia led to an increase in the diazoxide dose to $12.6 \mathrm{mg} / \mathrm{kg} /$ day associated with progressive worsening of diffuse hypertrichosis over the whole body including her face, trunk and limbs. Her appetite was poor and hence, if hypoglycaemic, required forced feeding, which led to excessive weight gain. The option of octreotide was discussed with both parents. Since octreotide is administered as a parenteral injection several times a day, with the possibility of tachyphylaxis, the parents were keen to proceed with a trial of oral sirolimus, even though the long-term side effects were not known. At 15 months of age, in view of the intermittent and persistent episodes of hypoglycaemia, she was commenced on oral sirolimus at a dose of $0.1 \mathrm{mg}$ tds $\left(0.5 \mathrm{mg} / \mathrm{m}^{2} /\right.$ day $)$, which was titrated to achieve a target level between 5 and $15 \mathrm{ng} / \mathrm{mL}$ with regular monitoring of her full blood count, liver and renal function tests. The sirolimus trough level was checked monthly and the dose was titrated upwards to $1.0 \mathrm{mg}$ tds $\left(4.4 \mathrm{mg} / \mathrm{m}^{2} /\right.$ day), which eventually maintained the serum sirolimus trough level between 25 and $30 \mathrm{ng} / \mathrm{mL}$, with abolition of hypoglycaemia. The diazoxide dose was concurrently decreased and stopped 3 months after starting sirolimus.

Sirolimus was well tolerated and the hypertrichosis resolved. She was feeding significantly better and the frequency of feeds was reduced, as her capillary glucose levels were generally above $4-5 \mathrm{mmol} / \mathrm{L}$. This resulted in less weight gain. However, she developed oral ulcers with clinically insignificant elevations in the serum CK to a maximum of $694 \mathrm{U} / \mathrm{L}$ (normal range: $20-300 \mathrm{U} / \mathrm{L}$ ) and ALT levels to a maximum of $83 \mathrm{U} / \mathrm{L}$ (20-65).

After 15 months of treatment with sirolimus, she was able to maintain her capillary glucose levels $>4 \mathrm{mmol} / \mathrm{L}$ for progressive longer periods of time and the borderline hypoglycaemic episodes only occurred when she was unwell with poor feeding and vomiting. As her capillary glucose levels were stable with no hypoglycaemic episodes, she was weaned off sirolimus over the next 6 months. There were no hypoglycaemic episodes when the sirolimus dose was tapered and her capillary glucose levels remained consistently $>4 \mathrm{mmol} / \mathrm{L}$. However, 1 month after stopping sirolimus, she developed a bout of viral gastroenteritis and was hospitalized for 5 days with severe vomiting, watery diarrhoea and poor feeding. Her lowest serum glucose level was $2.5 \mathrm{mmol} / \mathrm{L}$ and she was started on a $10 \%$ dextrose drip to maintain normoglycaemia. When she started to feed again, her blood glucose levels were maintained $>4.0 \mathrm{mmol} / \mathrm{L}$, the dextrose drip was stopped and she was discharged well. At her most recent outpatient visit, she was 3 years 3 months of age and her developmental milestones were normal. She was able to maintain her capillary glucose levels $>3.5 \mathrm{mmol} / \mathrm{L}$, even after 10 hours fasting. Both the serum CK and ALT levels normalized after stopping sirolimus.

\section{Discussion}

The management of diffuse $\mathrm{HH}$ to prevent recurrent hypoglycaemia and brain damage is very challenging. Conventional treatments including the use of diazoxide, octreotide and nifedipine are not always able to prevent hypoglycaemic episodes and are often associated with unpleasant side effects. Historically, severe cases of diffuse hyperinsulinism, which did not respond to medical treatment required near total pancreatectomy, result in diabetes mellitus and exocrine pancreatic insufficiency, with possible persistent hypoglycaemia after surgery. Recently, the use of sirolimus was introduced as a potential effective alternative medical treatment for congenital hyperinsulinism. The mTOR signalling pathway promotes $\beta$-cell growth, islet size and insulin production. This pathway is crucial for $\beta$-cell function and glycaemic control (6). The inhibition of the mTOR pathway by sirolimus can potentially cause suppression of $\beta$-cell proliferation and reduce hyperinsulinism.

This is the first report of successful treatment with sirolimus in a partially diazoxide-responsive $\mathrm{HH}$ child. Initial treatment with diazoxide resulted in a significant improvement in the capillary glucose levels and the child could be discharged from hospital on oral feeds. Not unexpectedly, there were occasional breakthrough episodes of mild hypoglycaemia not requiring any further admissions or escalation in therapy, which was in keeping with sudden surges of insulin release, as a consequence of the inactivating mutations of the sulphonylurea receptor. However, the diazoxide-associated side effects including poor appetite, excessive weight gain from overfeeding to treat hypoglycaemia and generalized hypertrichosis were very distressing for the family.

After conversion to sirolimus, there was significant improvement with complete resolution of hypoglycaemia when the trough sirolimus levels ranged from 25 to $30 \mu \mathrm{g} / \mathrm{L}$. This was higher than the original trough sirolimus level of $5-15 \mathrm{ng} / \mathrm{mL}$, as reported by Seniappan (7), but it was 
necessary in order to eliminate all the hypoglycaemic episodes while diazoxide was weaned off. The deceleration of weight gain with near complete resolution of the hypertrichosis are other important factors to consider. Potential side effects of sirolimus include mucositis, hyperlipidaemia, pneumonitis, severe infections and bone marrow suppression, which have been reported in adult studies and are reversible with dose reduction (8). In our patient, her blood counts remained normal throughout the treatment and there was no increased frequency of infections after starting sirolimus. The side effects noted were transient oral ulcers and clinically insignificant elevations in the serum CK and ALT. Oral ulcers after administration of sirolimus can be minimized by rinsing the mouth with water or juice.

Hyperinsulinism often becomes milder over time with slow progressive $\beta$-cell apoptosis in patients with diffuse HH. Animal studies have also demonstrated that rapamycin (also known as sirolimus) can cause a $50 \%$ reduction in $\beta$-cell mass by increased apoptosis (9). Since this patient's capillary glucose levels had been stable for 6 months and the long-term side effects of sirolimus are not known, sirolimus was gradually weaned off over 6 months. With this, the oral ulcers stopped appearing. The half-life of sirolimus is approximately $63 \mathrm{~h}$ and sirolimus should be eliminated from the body within 5 half-lives, or approximately 13.1 days. For this child, the capillary glucose levels were maintained $>4 \mathrm{mmol} / \mathrm{L}$, although she was recently hospitalized for a hypoglycaemic episode associated with severe viral gastroenteritis. Close monitoring will be necessary to ascertain if she can maintain long-term normoglycaemia without sirolimus.

In conclusion, sirolimus significantly improved this child's blood glucose levels, allowing cessation of diazoxide with resolution of hypertrichosis and prevention of excessive weight gain. This illustrates the utility of sirolimus in treating diffuse $\mathrm{HH}$, which is not fully responsive to diazoxide, while avoiding the untoward side effects of diazoxide. While transient elevations in serum CK and ALT levels were noted in our patient, more studies into the long-term efficacy and safety of sirolimus are necessary.

\section{Declaration of interest}

The authors declare that there is no conflict of interest that could be perceived as prejudicing the impartiality of the research reported.

\section{Funding}

This research did not receive any specific grant from any funding agency in the public, commercial or not-for-profit sector.

\section{Patient consent}

Written informed consent was obtained from the patient's mother for publication of the submitted article.

\section{Author contribution statement}

Loke Kah Yin is the primary physician responsible for the patient. Andrew Sng, Yvonne Lim and Cindy Ho assisted in managing the patient. Maria Güemes assisted in the preparation of the manuscript. Khalid Hussain provided the protocol and guidance with the use of sirolimus in treating congenital hyperinsulinism.

\section{Acknowledgements}

The authors thank Dr Dimple Rajgor for her assistance in submission of the manuscript for publication.

\section{References}

1 Flanagan SE, Kapoor RR \& Hussain K 2011 Genetics of congenital hyperinsulinemic hypoglycemia. Seminars in Pediatric Surgery 20 13-17. (doi:10.1053/j.sempedsurg.2010.10.004)

2 Pape L \& Ahlenstiel T 2014 mTOR inhibitors in pediatric kidney transplantation. Pediatric Nephrology 29 1119-1129. (doi:10.1007/ s00467-013-2505-9)

3 Alexandrescu S, Tatevian N, Olutoye O \& Brown RE 2010 Persistent hyperinsulinemic hypoglycemia of infancy: constitutive activation of the mTOR pathway with associated exocrine-islet transdifferentiation and therapeutic implications. International Journal of Clinical and Experimental Pathology 3 691-705.

4 Kulke MH, Bergsland EK \& Tao JC 2009 Glycemic control in patients with insulinoma treated with everolimus. New England Journal of Medicine 360 195-197. (doi:10.1056/NEJMc0806740)

5 Pratik S, Arya VB, Flanagan S, Morgan K, Ellard S, Sanniappan S \& Hussain K 2015 Sirolimus therapy in a patient with severe hyperinsulinaemic hypoglycaemia due to a compound heterozygous ABCC8 gene mutation. Journal of Pediatric Endocrinology and Metabolism 28 695-699. (doi:10.1515/jpem-2014-0371)

6 Mori H, Inoki K, Opland D, Munzberg H, Villanueva EC, Faouzi M, Ikenoue T, Kwiatkowski DJ, MacDougald OA, Myers MG Jr, et al. 2009 Critical roles for the TSC-mTOR pathway in $\beta$-cell function. American Journal of Physiology: Endocrinology and Metabolism 297 E1013-E1022. (doi:10.1152/ajpendo.00262.2009)

7 Senniappan S, Alexandrescu S, Tatevian N, Shah P, Arya V, Flanagan S, Ellard S, Rampling D, Ashworth M, Brown R, et al. 2014 Sirolimus therapy in infants with severe hyperinsulinemic hypoglycemia. New England Journal of Medicine 370 1131-1137. (doi:10.1056/ NEJMoa1310967)

8 Sankhala K, Mita A, Kelly K, Mahalingam D, Giles F \& Mita M 2009 The emerging safety profile of mTOR inhibitors, a novel class of anticancer agens. Targeted Oncology 4 135-142. (doi:10.1007/s11523009-0107-z)

9 Yang SB, Lee HY, Young DM, Tien AC, Rowson-Baldwin A, Shu YY, Jan YN \& Jan LY 2012 Rapamycin induces glucose intolerance in mice by reducing islet mass, insulin content and insulin sensitivity. Journal of Molecular Medicine 90 575-585. (doi:10.1007/s00109-011-0834-3)

Received in final form 14 September 2016

Accepted 22 September 2016 